Research Article

\title{
Peculiarities of Functional Connectivity-including Cross-Modal Patterns_-in Professional Karate Athletes: Correlations with Cognitive and Motor Performances
}

\author{
Beatrice Berti, ${ }^{1,2}$ Davide Momi, ${ }^{1,3,4}$ Giulia Sprugnoli, ${ }^{1}$ Francesco Neri, ${ }^{1}$ Marco Bonifazi, ${ }^{2}$ \\ Alessandro Rossi, ${ }^{1}$ Maria M. Muscettola, ${ }^{2}$ Roberto Benocci, ${ }^{5}$ Emiliano Santarnecchi $\mathbb{D}^{1,3,6}$ \\ and Simone Rossi $\mathbb{D D}^{1,2}$ \\ ${ }^{1}$ Siena Brain Investigation \& Neuromodulation Lab (Si-Bin Lab), Department of Medicine, Surgery and Neuroscience, Neurology and \\ Clinical Neurophysiology Section, University of Siena, Italy \\ ${ }^{2}$ Department of Medicine, Surgery and Neuroscience, Human Physiology Section, University of Siena, Italy \\ ${ }^{3}$ Berenson-Allen Center for Non-Invasive Brain Stimulation, Beth Israel Medical Center, Harvard Medical School, Boston, MA, USA \\ ${ }^{4}$ Department of Neuroscience, Imaging and Clinical Sciences, University of Chieti-Pescara, Chieti, Italy \\ ${ }^{5}$ ASD Shinan Karate Kai of Siena, Siena, Italy \\ ${ }^{6}$ Siena Robotics and Systems Lab (SIRS-Lab), Engineering and Mathematics Department, University of Siena, Italy
}

Correspondence should be addressed to Simone Rossi; simone.rossi@unisi.it

Received 7 October 2018; Revised 4 April 2019; Accepted 26 May 2019; Published 26 June 2019

Academic Editor: Guy Cheron

Copyright ( 2019 Beatrice Berti et al. This is an open access article distributed under the Creative Commons Attribution License, which permits unrestricted use, distribution, and reproduction in any medium, provided the original work is properly cited.

Professional karate is a sport activity requiring both physical and psychological skills that have been associated with a better "global neural efficacy." By means of resting state functional magnetic resonance imaging (rs-fMRI), we investigated the neural correlates of cognitive and kinematic abilities in a group of 14 professional karateka and 14 heathy matched controls. All subjects underwent an extensive cognitive test battery for the identification of individual multidimensional cognitive profile and rs-fMRI scans investigating functional connectivity (FC). Moreover, kinematic performances in athletes were quantified by the Ergo-Mak, an integrated system developed for measuring motor reactivity, strength, and power of athletic gestures. Karateka performed significantly better than controls in the visual search task, an ability linked with increased positive correlations in FC between the right superior parietal lobe and bilateral occipital poles. Kinematic performances of athletic feats were sustained by increased positive correlations between subcortical (cerebellum and left thalamus) and cortical (inferior frontal cortex, superior parietal cortex, superior temporal cortex) regions. An unexpected FC increase between auditory and motor-related areas emerged in karateka, possibly reflecting a cross-modal coupling due to the continuous exposure to either internal or external auditory cues, positing this sensory channel as a possible target for novel training strategies. Results represent a further step in defining brain correlates of "neural efficiency" in these athletes, whose brain can be considered a model of continuous plastic train-related adaptation.

\section{Introduction}

The martial art of karate includes two different disciplines: kumite and kata. The former consists of battling and combat exercises with the antagonist; the latter is characterized by various offensive and defensive postures realized without any opponent. In both cases, a strong and deep vocalization, called kiai, accompanies the execution of a given technique (see Supplementary materials and Video 1 for details).

At the professional level, these karate disciplines require an uncommon combination of perceptual (visual, proprioceptive, auditory, and interoceptive) [1,2], cognitive (working memory, attention, and executive functioning) $[3,4]$, and motor (sprint, reactivity, coordination ability, and explosive 
and strong ballistic motor skills) abilities [1, 5-11] that are progressively refined and improved with continuous training, especially in terms of processing speed and execution timing. On the basis of electroencephalographic (EEG) [12-15] and diffusor tensor imaging (DTI) investigations [16], it has been postulated that such behavioural efficiency in karate athletes is paralleled by a better "neural efficiency," that is, an optimized communication between different recruited brain regions.

The neural efficiency hypothesis describes the phenomenon for which brighter individuals show lower brain activation compared to less bright individuals when working on the same cognitive tasks [17]. Several studies have demonstrated this concept in nonathletes too, showing that subjects with higher intelligent quotient who outperform in word fluency, spatial skills, and working memory tests were characterized by lower cortical activations [18-21]. Such evidences have been recently extended to investigate the cortical processes of elite experts or athletes during the specific challenges in which they were highly practiced. Specifically, more focal as well as more efficiently organized neural activation patterns have been reported in expert golfers [22], elite archers [23], professional race car drivers [24], professional footballers [25], and musicians [26].

Moreover, evidences suggest that experts differ in visuospatial abilities directly tied to their domain of expertise. For instance, recent studies have reported quantitative differences in brain activation during visuospatial processing in elite rugby players [27] and archery experts [28], compared to novices, supporting the existence of a specific sportrelated cognitive strategy.

Here, we aimed to directly address the functional connectivity (FC) patterns of brain networks during resting state functional magnetic resonance imaging (rs-fMRI) in professional karate athletes compared to matched nonathlete controls. To our knowledge, no similar investigation has so far been conducted, despite its potential relevance for understanding the neural underpinnings of exceptional multimodal abilities, neural efficiency, and brain plastic adaptation to extensive karate training. To better address these multimodal features in relation to the connectivity changes, we also attempted to correlate whole brain FC indexes with kinematic, dynamic, and psychoergonomic features of the athletic performance, as recorded and quantified by means of "Ergo-Mak," a tool developed specifically to capture reaction times, speed, accuracy, and power indexes from any voluntary visually/acoustically triggered athletic gesture.

Resting state FC has been successfully used to probe the brain's functional pattern in healthy subjects [29-35], as well as in neurological and psychiatric patients [36-39]. However, the effects of professional training on functional brain networks' patterns have not yet been studied in the karate discipline context.

We hypothesized that the peculiar perceptual, cognitive, and mostly motor skillful abilities of karate athletes would rely on particularly evident cross-modal patterns of FC encompassing attentional, visual, and possibly auditory networks, as well as cortical and subcortical structures involved in coordination, timing, and maintenance of a sequence of actions, which may play an important role in determining the accuracy, speed, and strength of a karateka feat. The identification of such networks opens the possibility to improve professional training and athletic performance.

\section{Subjects and Methods}

2.1. Participants and Experimental Design. The study was carried out on 14 experienced top-level karateka, 8 males and 6 females (mean age: $23.7 \pm 4.1$ years), belonging to the Shotokan hard style (ASD Shinan Karate Kai, Siena, Italy) and 14 age- and sex-matched participants as the control group (mean age $24 \pm 2.9$; 10 males and 4 females). All subjects were right-handed, based on the handless questionnaire [40]. The athletes had been engaged in karate for more than four years (mean: $14 \pm 6.8$ years): twelve were black belt (three were 1st dan, five were 2nd dan, and four were 3rd dan) and two brown belt. Ten of the black belts practiced kumite and were all at the competitive level of the Italian championships. Before the beginning of the experiment, all subjects signed an informed-consent form and filled in a questionnaire concerning their habits, health, diet, sleep, smoking, use of drugs, alcohol and caffeine, sport activity, and work. All subjects were familiar with computers, but they were not skilled in videogames or other computer-ability performance, which could have affected reaction times. In order to get a complete cognitive profile of the participants, a screening visit was performed, where a vast cognitive battery was administered and magnetic resonance imaging performed (Figure 1). All measures (cognitive, fMRI, and kinematic) were acquired in the same time period. Only the karate group's and control subjects in good health, free of drugs and medications, and with negative psychiatric, neurological, and endocrine histories were enrolled in the study. The experiment complied with the current Italian law and the Helsinki Declaration of the World Medical Association, and it was approved by the Local Ethic Committee.

2.2. Assessment of Motor Performance and Kinematics Data: The Ergo-Mak Integrated System (See Supplementary Materials, Video 1). The "Ergo-Mak" integrated system (ASD Shinan Karate Kai, Siena, Italy; patent no. SI2004U000001) is employed to record kinematic, dynamic, and psychoergonomic movement parameters of the athletic feat. Karateka stand in bare feet on the centre of a force plate with their feet separated by $10 \mathrm{~cm}$. Athletes are asked to punch a vertical force plate placed on the Ergo-Mak machine, which records the movement parameters including reaction times, strength, and efficacy of the gesture. The subject's hand is connected by a rigid cord to a revolving transducer positioned behind the athlete, which is used to measure the time of the strike before it reaches the target. Overall, it allows us to measure the position, speed, and acceleration of the hand. Furthermore, a $10 \mathrm{~mm}$ thick rubber strip was placed directly behind the surface of impact of the plank, which was attached to a high tech linear transducer used to measure the dynamic parameters of impact. The magnetic strip of the linear transducer was used to measure power, strength, and work (Figure 2(a) and Supplementary materials). 

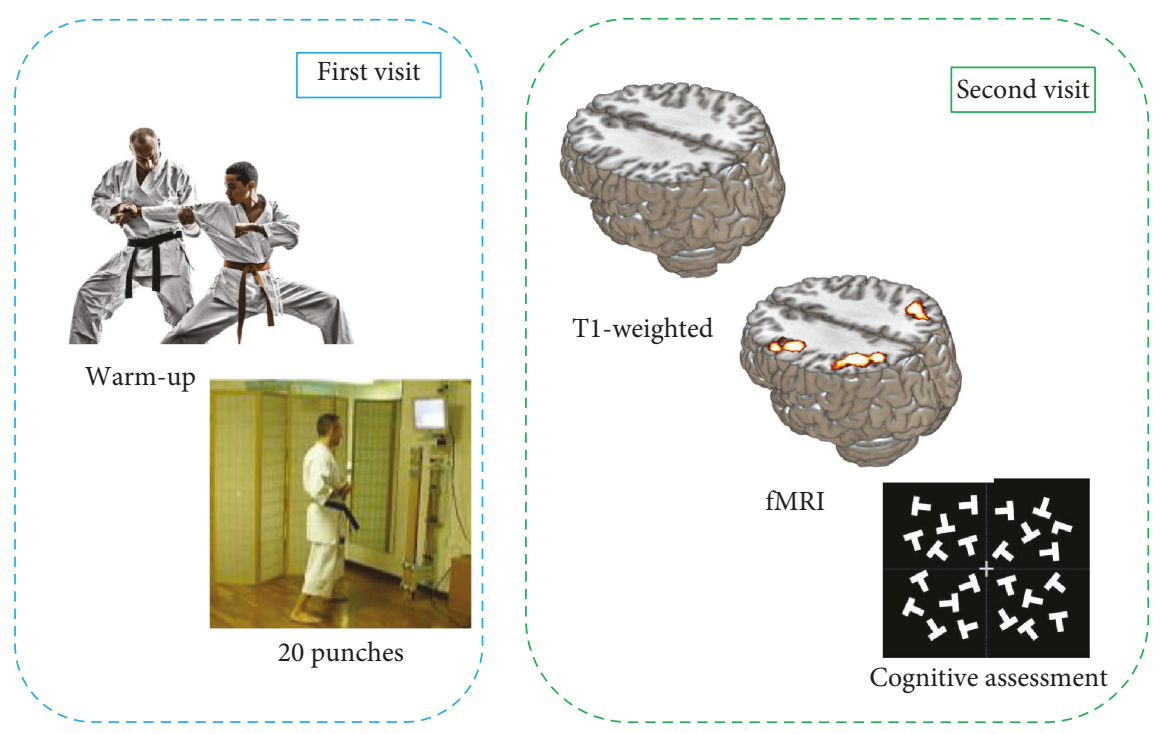

(a)



(b)
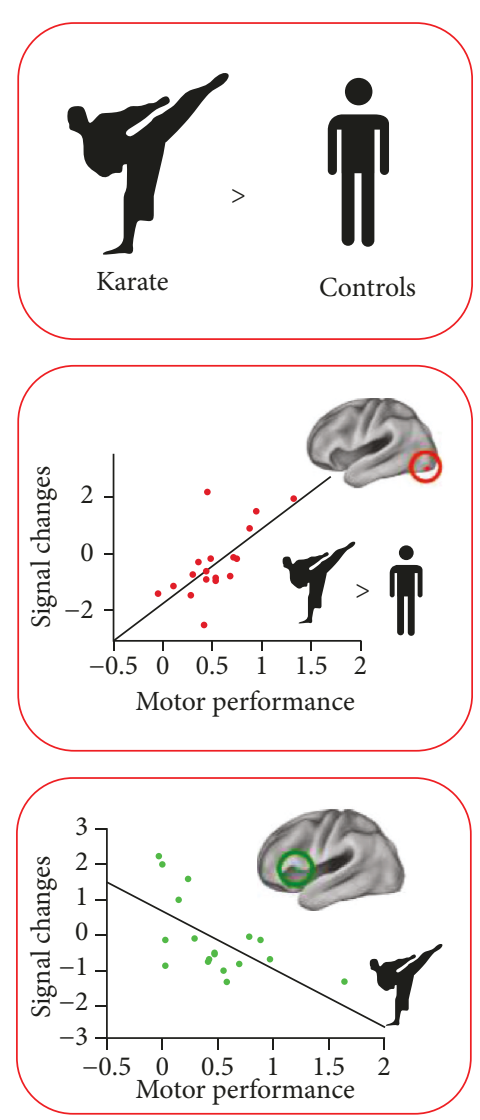

(c)

FIgURE 1: Study overview. Schematic representation of the experimental timeline (a), statistical analyses (b), and contrasts (c) (see supplemental material).

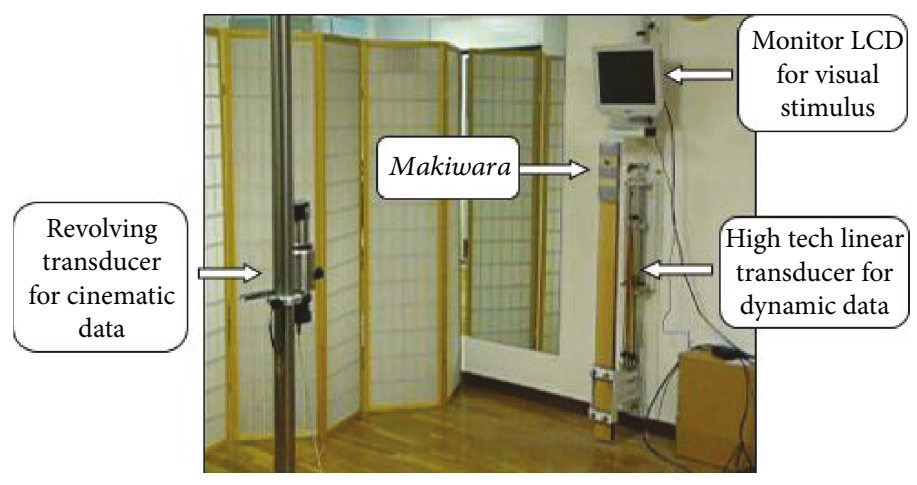

(a)

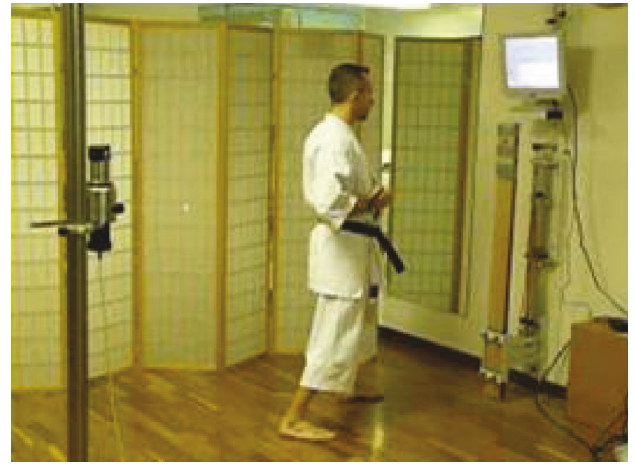

(b)

FIGURE 2: The "Ergo-Mak" integrated system used to record kinematic, dynamic, and psychoergonomic movement parameters of the athletic feat. Details of measurements are in the text (see Supplementary materials and video 1).

After 10 minutes of warm-up, each karateka performed a series of 20 frontal karate punches (gyaku tsuki) with their right arm (dominant arm for all subjects). Before the beginning of the motor action, the subject stood at a 1-meter distance in front of a computer monitor screen, in a typical karate position before action (gedan barai), with his hand connected by a linear encoder positioned behind him
(Figure 2(b)). Each gyaku tsuki was executed as rapidly as possible to impact the target at full strength with the metacarpo-phalangeal joint and part of the phalange of the second and third finger. The target (makiwara) was a flexible plank made of the following materials: wood, fibreglass, and carbon fibre. After each gyaku tsuki, the subject repositioned his hand. Each trial consisted of a period of 8.0 seconds, 
starting with a warning stimulus, a ringing noise, followed by a premotion random period lasting from 2.0 to 6.0 seconds until the onset of an imperative stimulus. The interval between trials was 2.0 seconds. In this period, the subject had to stay still and avoid any kind of movement. He then had to start the motor action as soon as possible when the imperative stimulus, a red rhombus, appeared on the computer screen. Physiological data were recorded throughout the duration of the trial and then collected and analysed.

2.3. Cognitive Battery (Baseline Assessment). Subjects underwent a full cognitive battery (120 minutes) to capture their individual cognitive profiles (Figure 1(a), Supplementary materials 2.1 and 2.2). Baseline assessment consisted of ten short tasks covering the cognitive domains of attention, fluid intelligence, inhibition, verbal and visuospatial working memory, and switching flexibility. The cognitive battery included the following tasks: global-local (GL) features, preparing to overcome prepotency (POP) letter no-go (LNG), change localization (CL), visual search (VS), serial reaction time task (SRTT), mental rotation (MR), attentional blink (AB) task, flanker task (FT), enumeration (ET), and useful field of view (UFOV). Cognitive assessment was performed on a Windows laptop PC (Microsoft) equipped with E-Prime 2.0 software (Psychology Software Tools Inc.; http://www.pstnet.com/eprime).

2.4. Magnetic Resonance Imaging (MRI) Data Acquisition. Imaging was performed on a Philips Intera 1.5T MRI scanner. A T1-weighted anatomical image and two rs-fMRI sessions (TR/TE 2500/40 ms, 200 scans, 23 slices, total scanning length $8 \mathrm{~min}$ ) were acquired for each participant in a rest condition during the first visit. Subjects were asked to remain as still as possible and awake in the scanner, with their eyes open.

SPM8 software (Statistical Parametric Mapping; http:// www.fil.ion.ucl.ac.uk/spm/) was used for the preprocessing steps of the acquired images. The first three volumes of functional images were discarded for each subject to allow for steady-state magnetization. EPI images were slice-time corrected using the interleaved descending acquisition criteria and realigned and resliced to correct for head motion using a mean functional volume derived from the overall fMRI scans. A matrix for each subject had been used during the statistical analysis to completely remove the effects of these timepoints. Subjects whose head motion exceeded $1.0 \mathrm{~mm}$ or rotation exceeded $1.0^{\circ}$ during scanning were excluded (see the Supplementary materials for further details on the preprocessing pipeline).

\section{Statistical Analysis}

3.1. Behavioural Performance. Accuracy and reaction time changes were evaluated for each test of the cognitive battery using the Statistical Package for the Social Sciences (SPSS) Version 20 [41]. A one-way ANOVA with the factor Group (between subjects) was used to test the existence of statistical significance differences in karate vs. control comparisons. Bonferroni post hoc analysis was applied to the performance scores, lowering the significant threshold to $p=0.002$. Means, standard deviations, and $p$ values for all the tasks are summarized in Table 1.

3.2. Motor Performance. Reaction times, speed, and power of each motor action were recorded and collected by means of the Ergo-Mak integrated system (see video 1 and Supplementary materials 2.3).

Reaction times refer to the speed at which a person moves in response to a stimulus, i.e., the degree of reactivity of a motor action [1, 2, 11]. The Ergo-Mak measured reaction times through the revolving transducer positioned behind the athlete and connected by the rigid cord to the hand of the karateka. Reaction times were measured in milliseconds between the appearance of the visual stimulus (red rhombus) and the time when the hand started to move. Ergo-Mak also provided the reading of the position of the hand (measured in meters) during the punch before it reached the target with a sampling frequency of 5000 Hertz. This allowed measures of maximum, contact, and average speed of the hand during the entire duration of each motor action.

As soon as the hand impacted the makiwara, the high tech linear transducer, positioned directly behind the plank, measured the following dynamic data: strength, energy, and power. The speed of the hand at the moment of impact is directly proportional to the strength of a gyaku tsuki indexing a variation of speed of the makiwara. Therefore, it allowed calculation of energy transfer at the time of the impact. Furthermore, the speed at which the makiwara moved in response to the impact was directly correlated with the power of the technique.

A principal component analysis (PCA) [42] was employed to convert the set of motor performance measures into a set of 3 uncorrelated variables (strength, power, and execution).

3.3. Resting State Functional Connectivity (rs-FC). CONNfMRI functional connectivity toolbox v15g (http://www. nitrc.org/projects/conn) [43] was implemented in MATLAB for the analyses of rs-FC (Figure 1(b) reports the methods used for rs-FC analysis). The Harvard-Oxford (cortical and subcortical) atlas and the Shirer network-based atlas (Shirer et al. (2012) http://findlab.stanford.edu/functional_ROIs, Stanford University, Palo Alto, CA) were employed for rs-FC analysis providing different parcellations of the same brain. Specifically, the former accounts for a whole brain measure whist the latter provides a network-based measure. Even though most of the studies on rs-FC implemented only one of those atlases, we decided to employ both of them in order to investigate results both at the anatomical and network levels.

Region of interest (ROI) and seed-to-voxel analyses were conducted as second-level analyses to identify potential differences in karate vs. control comparisons. Age, gender, and year of karate experience (in the case of the karate group) were entered as second-level covariates of no interest.

The ROI-to-ROI analysis allowed to pinpoint significant FC changes using two-sided comparisons $(p<0.05$ false discovery rate (FDR) corrected) [44]. Through the use of 
TABLE 1: Performance of athletes and control group subjects for the cognitive tasks.

\begin{tabular}{|c|c|c|c|c|c|c|}
\hline \multirow{3}{*}{$\begin{array}{l}\text { Enumeration } \\
\end{array}$} & \multicolumn{2}{|c|}{ Karate group } & \multicolumn{2}{|c|}{ Control group } & \multicolumn{2}{|c|}{ Karate vs control } \\
\hline & \multirow[t]{2}{*}{ Mean } & \multirow[t]{2}{*}{ SD } & \multirow[t]{2}{*}{ Mean } & \multirow[t]{2}{*}{ SD } & \multirow[t]{2}{*}{$F$} & \multirow[t]{2}{*}{$p$ value } \\
\hline & & & & & & \\
\hline ACC & 0.46 & 0.11 & 0.55 & 0.1 & 5.275 & $0.03^{*}$ \\
\hline RTs & 1704.53 & 641.43 & 1986.26 & 591.05 & 1.461 & 0.238 \\
\hline \multicolumn{7}{|l|}{ Mental rotation } \\
\hline ACC & 0.79 & 0.07 & 0.79 & 0.08 & 0.075 & 0.786 \\
\hline RTs & 1058.51 & 293.45 & 965.41 & 312.91 & 0.66 & 0.424 \\
\hline \multicolumn{7}{|c|}{ Useful field of view } \\
\hline ACC & 0.48 & 0.17 & 0.54 & 0.15 & 0.896 & 0.352 \\
\hline RTs & 610.35 & 260.54 & 599.42 & 184.99 & 0.016 & 0.899 \\
\hline \multicolumn{7}{|l|}{ Visual search } \\
\hline ACC & 0.92 & 0.03 & 0.94 & 0.01 & 1 & 0.327 \\
\hline $\mathrm{RT}$ & 1772.34 & 200.23 & 2101.18 & 299.32 & 11.674 & $0.002^{* *}$ \\
\hline \multicolumn{7}{|c|}{ Global-local features } \\
\hline GLO ACC & 0.96 & 0.04 & 0.97 & 0.02 & 0.293 & 0.593 \\
\hline GLO RTs & 792.07 & 332.56 & 1104.5 & 292.84 & 1.249 & 0.274 \\
\hline LOC ACC & 0.95 & 0.05 & 0.96 & 0.04 & 0.356 & 0.321 \\
\hline LOC RTs & 813.85 & 344.94 & 1109.1 & 320.56 & 1.359 & 0.256 \\
\hline \multicolumn{7}{|c|}{ Preparing to overcome prepotency } \\
\hline ACC & 0.97 & 0.01 & 0.96 & 0.003 & 1.557 & 0.223 \\
\hline RTs & 371.8 & 60.90 & 423.89 & 95.30 & 2.969 & 0.097 \\
\hline \multicolumn{7}{|l|}{ Flanker } \\
\hline ACC & 0.7 & 0.07 & 0.74 & 0.07 & 3.093 & 0.09 \\
\hline RTs & 432.93 & 138.99 & 514.1 & 135.35 & 2.451 & 0.13 \\
\hline \multicolumn{7}{|c|}{ Change localization } \\
\hline ACC & 0.73 & 0.15 & 0.77 & 0.15 & 4.218 & 0.05 \\
\hline RTs & 1858.61 & 548.15 & 2110.71 & 641.96 & 3.337 & 0.079 \\
\hline \multicolumn{7}{|c|}{ Serial reaction time task } \\
\hline ACC & 0.95 & 0.05 & 0.96 & 0.03 & 1.345 & 0.257 \\
\hline RTs & 218.57 & 42.37 & 209.41 & 34.36 & 0.395 & 0.535 \\
\hline \multicolumn{7}{|l|}{ Letter no-go } \\
\hline ACC & 92.8 & 3.87 & 85.71 & 12.23 & 1.557 & 0.223 \\
\hline RTs & 273.67 & 13.65 & 284.5 & 17.49 & 2.969 & 0.097 \\
\hline \multicolumn{7}{|c|}{ Attentional blink } \\
\hline Target ACC & 0.95 & 0.04 & 0.97 & 0.02 & 4.352 & $0.047^{*}$ \\
\hline Target RTs & 1176.31 & 343.51 & 1198.9 & 247.17 & 0.04 & 0.843 \\
\hline Xpresent ACC & 0.75 & 0.09 & 0.78 & 0.1 & 0.458 & 0.505 \\
\hline Xpresent RTs & 569.79 & 268.11 & 602.13 & 207.8 & 0.127 & 0.724 \\
\hline
\end{tabular}

network-based statistics [45], the significant nodes were taken into account for the seed-to-voxel analyses, which were performed using two-sided comparison with a voxelwise threshold level of $p<0.05$ uncorrected and a cluster-level threshold of $p<0.05$ FDR corrected.

The main goal of the study was to examine changes in rsFC profiles between karateka and control individuals. To this purpose, a one-way ANOVA analysis was implemented contrasting karate vs. controls, where group, age, and gender were entered as second-level nuisance covariates.
In a second step, whenever significant changes were detected in cognitive performances, these were entered in the analysis as second-level covariates of interest. The aim was to track a consistent rs-FC pattern in karate vs control contrasts, which would provide a linear explanation for the significantly better performance due to karate exposure.

Finally, motor performance indexes were extracted entering the three most correlated measures (full speed makiwara, average speed hand, and total time) for each principal component (Power, Speed, and Execution) as second-level 


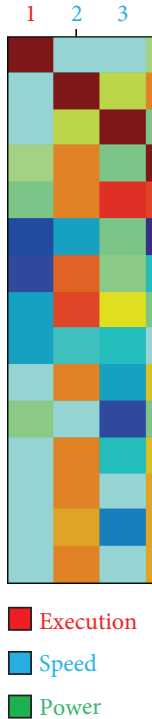

(a)
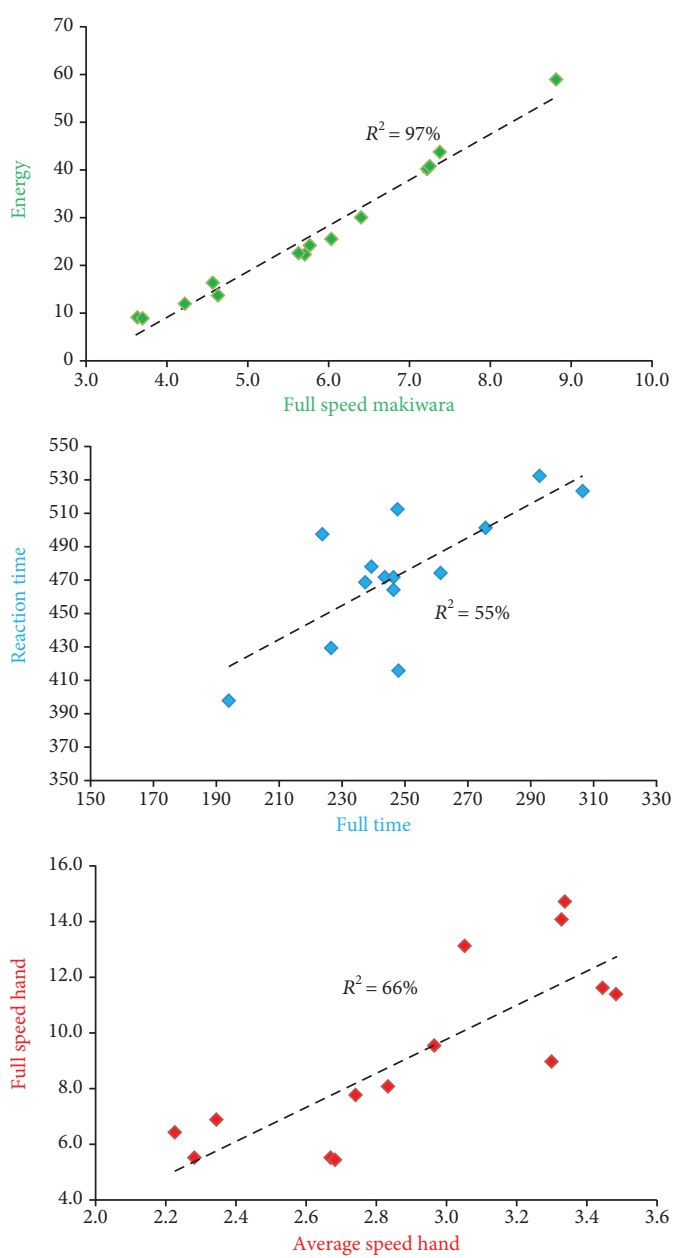

(b)

FIGURE 3: Statistical relationships involving motor performance measures. (a) Results of principal component analysis (PCA) showed in the form of a correlation matrix. The $82.86 \%$ of the total variance was explained by 3 latent variables defined as Power (green), Speed (blue), and Execution (red). (b) Scatter plots of the most correlated motor performance measures for the Power $\left(R^{2}=0.97\right)$, Speed $\left(R^{2}=0.55\right)$, and Execution $\left(R^{2}=0.66\right)$ principal components.

covariates. A multiple regression within-subject contrast was used for the karate group to examine whether the rs-FC patterns could explain the motor performance. Then, the voxels that significantly explain the motor performance were used as a mask to extract the FC strength. A scatter plot showing the correlation is shown.

\section{Results}

4.1. Behavioural Results at the Cognitive Battery. As shown in Table 1, significant differences in reaction time (RT) performance scores were found only for visual search $(F=11.674, p=0.002)$. Specifically, karate athletes showed faster RTs (mean difference $=328.84 \mathrm{~ms}$ ) without any significant difference in accuracy compared to the control group $(F=1.0, p=0.32)$.

With regard to the enumeration performance, a marginally significant $p$ value for accuracy emerged $(F=5.275, p=0.03)$, which however did not survive to the
Bonferroni correction for multiple comparisons, regardless of RT changes.

For the attentional blink task, there was a trend for higher accuracy in the identification of the first target $(F=4.352$, $p=.04$ ) but no difference in RTs. No significant changes in performance were shown in response to the second target, both for accuracy and RTs.

Likewise, no significant changes in performance were reported for MR, UFOV, GL, FLA, CL, SRTT, LNG, and POP.

4.2. Motor Performance (Karate Athletes Only). A principal component analysis (PCA) was applied in trying to reduce the complex kinematic dataset to a lower dimension (Figure 3). A set of three linearly uncorrelated variables (Power, Speed, and Execution) was extracted, which explained $82.86 \%$ of the total variance. These variables were then used for correlations with the fMRI results.

4.3. Resting State Functional Connectivity Analyses. As shown in Figure 4, ROI-to-ROI analyses for rs-fMRI revealed 


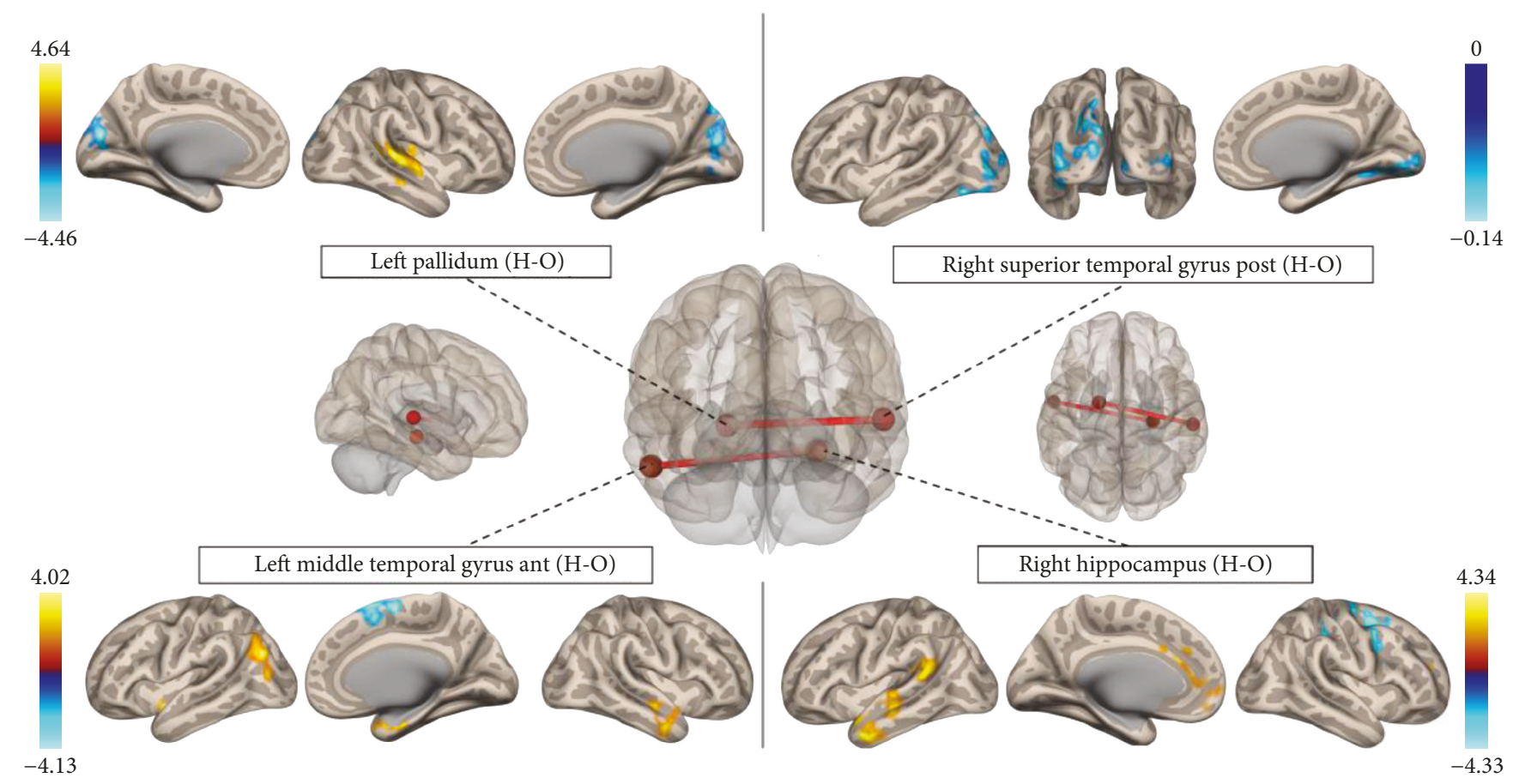

FIGURE 4: Resting state functional connectivity differences between groups. ROI-to-ROI analyses revealed a positive correlation in the left pallidum-right superior temporal gyrus post and right hippocampus-left middle temporal gyrus ant connections (centre). Increase (red) or decrease (blue) in seed-to-voxel connectivity maps for each node is shown for the threshold ( $p<0.05$, FDR corrected). An unthresholded pattern activation is shown in Figure S2, supplementary materials. All voxels with significant connectivity changes are shown in the figure and listed in Table S1, supplementary materials. Note: H-O: Harvard-Oxford.

significantly increased positive correlations for karate vs. control comparison in the left pallidum-right superior temporal gyrus posterior (post) $(t=4.67$, p-uncorrected $=$ $0.0001, \mathrm{p}-\mathrm{FDR}=0.01)$ and in the right hippocampus-left middle temporal gyrus anterior $(t=4.16, \mathrm{p}$-uncorrected $=$ $0.0004, \mathrm{p}-\mathrm{FDR}=0.038)$ connections. Seed-to-voxel analyses were further carried out taking each of these 4 nodes. When the left pallidum was taken as the seed, a stronger connectivity with a large cluster of voxels in the right superior and middle temporal gyri $(k=957 ; 70,-32,8)$ emerged, as well as a negative correlation within occipital regions ( $k=1340 ; 4,-88,28)$, such as the left and right cuneal cortex, left lateral occipital cortex, and left occipital pole.

When the right superior temporal gyrus was taken as the seed, a weaker connectivity with two clusters of voxels in the left $(k=1805 ;-36,-98,4)$ and the right $(k=804 ; 32,-82,2)$ occipital cortex was reported. For the right hippocampus, seed-to-voxel analysis showed instead a positive correlation with 3 different clusters belonging to cortical and subcortical regions, such as the cingulate gyrus $(k=1110 ;-2,30,28)$, the left middle temporal gyrus $(k=795 ;-52,2,-38)$, and the left supramarginal gyrus $(k=593 ;-62,-46,16)$. A negative correlation with a cluster in the right superior frontal gyrus $(k=914 ; 38,-2,44)$ also emerged.

Finally, seed-to-voxel analysis considering the left middle temporal gyrus anterior as the seed revealed an increase in connectivity with the right occipital cortex $(k=677 ;-46$, $-72,26)$ and the right $(k=1617 ; 24,-30,2)$ and the left $(k=792 ;-34,-44,-6)$ hippocampus, as well as a negative correlation with a cluster of voxels in the right superior frontal gyrus $(k=828 ;-4,-2,54)$ and crus I of the cerebellum $(k=709 ; 46,-68,-36)$. (See Supplementary Materials $1-4$ for additional details).

4.4. Correlations between Cognitive Profiles and FC Measure. Taking into account RTs for visual search (Figure 5), ROI-toROI analyses for karate vs. control contrast revealed a positive correlation between the right superior parietal lobule and the left $(t=4.28, \mathrm{p}$ - uncorrected $=0.0004$, and $\mathrm{p}-\mathrm{FDR}=0.02)$ and right $(t=4.18, \mathrm{p}-$ uncorrected $=$ 0.0005 , and $\mathrm{p}-\mathrm{FDR}=0.02$ ) occipital poles. Seed-to-voxel analysis was therefore carried out considering these three significant nodes. When the right superior parietal lobule was taken as the seed, an increase in connectivity was found with the bilateral occipital cortex $(k=10346 ; 12,-60,-16)$, the supplementary motor area $(k=711 ; 12,-4,44)$, and the right $(k=1239 ; 54,2,34)$ and left $(k=1078 ;-48,-18,6)$ precentral as well as postcentral sulci.

Once the left occipital lobe was considered as the seed for the analyses, a positive correlation with a cluster of voxels belonging to the right superior parietal lobule $(k=1585 ; 24,-52,72)$, right temporal cortex $(k=1097$; $24,-14,20)$, and left postcentral gyrus $(k=757 ;-22$, $-36,52)$ emerged.

Finally, when the right occipital lobe was taken as the seed, an increase in the connectivity with the left occipital 


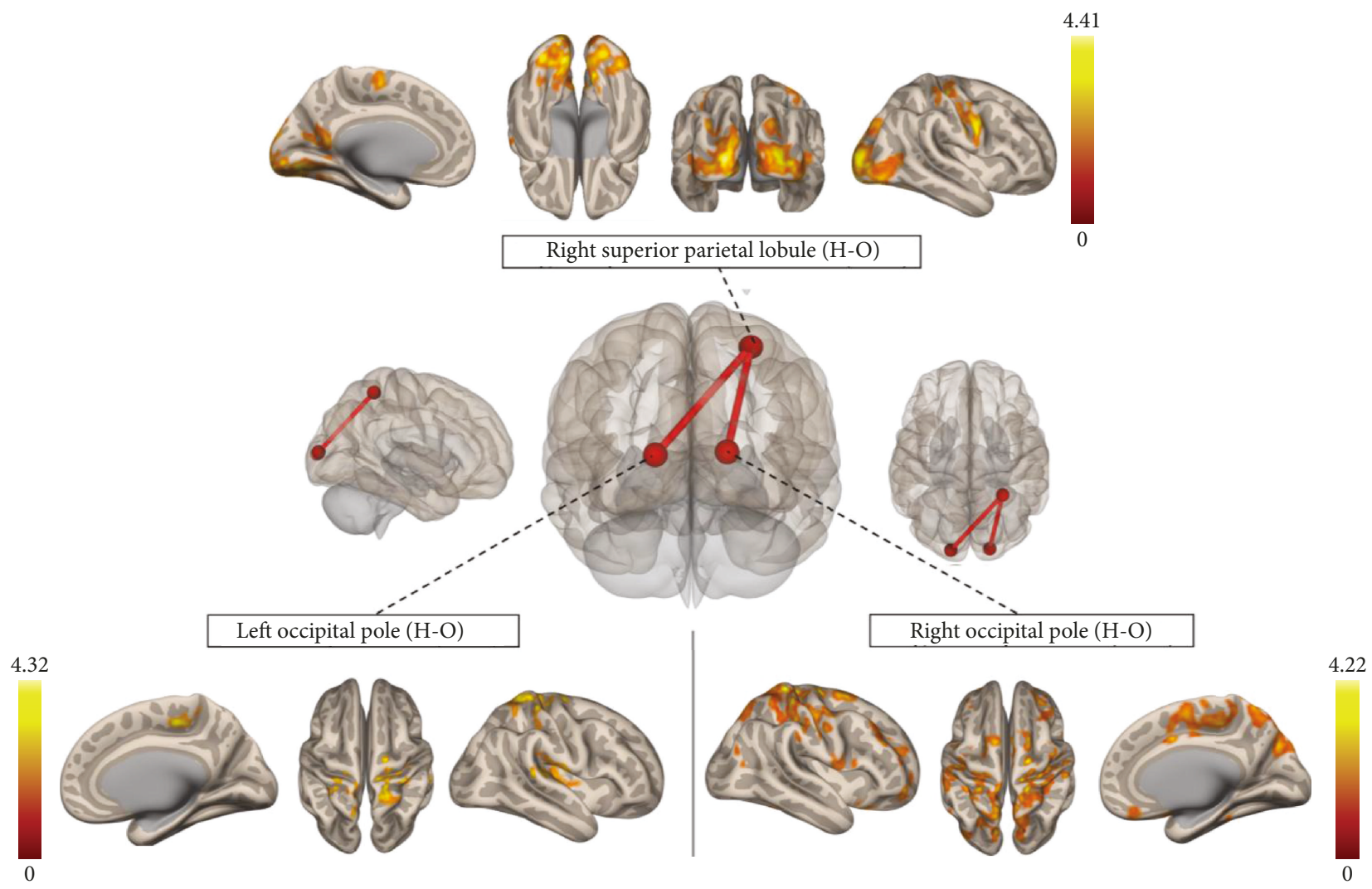

FIGURE 5: Visual search reaction time performance explained by resting state functional connectivity. ROI-to-ROI analyses revealed an increase in the right superior parietal lobule and bilateral occipital pole pathway, which may account for the better performance of the karate group (centre). Seed-to-voxel results, thresholded ( $p<0.05$, FDR corrected), are shown for each of the 3 nodes. Unthresholded pattern activation is shown in Figure S3 supplementary materials. All voxels with significant connectivity changes are shown in the figure and listed in Table S2, Supplementary materials. Note: H-O: Harvard-Oxford.

cortex $(k=1247 ;-10,-78,30)$, the right frontal pole $(k=676$ ; 32, 38, 48), the bilateral precentral and postcentral gyri $(k=9274 ; 44,00,56)$, and the left crus I $(k=635 ;-18,-60$, -22) was shown.

4.5. Correlations between Motor Performances and FC Measures. Considering "full speed makiwara" (the most representative Power factor in the PCA), ROI-to-ROI analyses revealed a hyperconnectivity between the left thalamus and the frontoparietal brain regions, including the left middle/superior frontal gyrus $(t=5.13, \mathrm{p}-$ uncorrected $=$ 0.0002 , and $p-F D R=0.02)$ and the left superior/inferior parietal lobule $(t=4.56, \mathrm{p}$ - uncorrected $=0.0007$, and $\mathrm{p}-\mathrm{FDR}=0.02) \quad($ see Figure 6$)$. Moreover, a positive correlation between the left inferior temporal cortex and the left inferior frontal gyrus $(t=4.92, \mathrm{p}$ - uncorrected $=0.0004$, and $\mathrm{p}-\mathrm{FDR}=0.02$ ) was found.

Considering the left thalamus as the seed, analyses revealed a negative correlation with the bilateral occipital pole $(k=4557 ; 24,-96,32)$, as well as an increase in connectivity with the left superior frontal cortex $(k=3270 ;-12,52,42)$, precuneus $(k=2141 ;-4,-72,30)$, right frontal pole $(k=674 ; 6,56,30)$, and the left $(k=757$; $-44,-62,32)$ and the right $(k=944 ; 36,-58,44)$ lateral occipital cortex. When the left middle/superior frontal gyrus was taken as the seed, a decrease in connectivity was observed with a cluster of voxels in the precuneus $(k=1022 ;-2,-24,34)$.

On the other hand, considering the left inferior frontal gyrus as the seed, an increase in connectivity with the left $(k=729 ;-50,-70,-4)$ and the right $(k=931 ; 52,-72,-14)$ lateral occipital cortex, as well as a negative correlation with a cluster of voxels in the precuneus $(k=844 ;-10,-50,30)$, was proved.

No suprathreshold voxels were found once taking either the left middle-occipital cortex or the left superior/inferior parietal lobule as the seed for the analyses.

Considering the "average speed hand" (the most representative Speed factor in the PCA), ROI-to-ROI analyses revealed an increased connectivity between the left lobule VIII-VIIb of the cerebellum and the bilateral temporal cortex, such as the right $(t=4.95, \mathrm{p}-$ uncorrected $=0.0003$, and $\mathrm{p}-\mathrm{FDR}=0.03)$ and the left $(t=4.30, \mathrm{p}-$ uncorrected $=0.001$, and $\mathrm{p}-\mathrm{FDR}=0.04$ ) supramarginal gyrus (see Figure 7).

When the left lobule VIII-VIIb of the cerebellum was taken as the seed, analyses revealed a positive correlation with the bilateral frontal pole $(k=2727 ;-34,52,10)$, the 


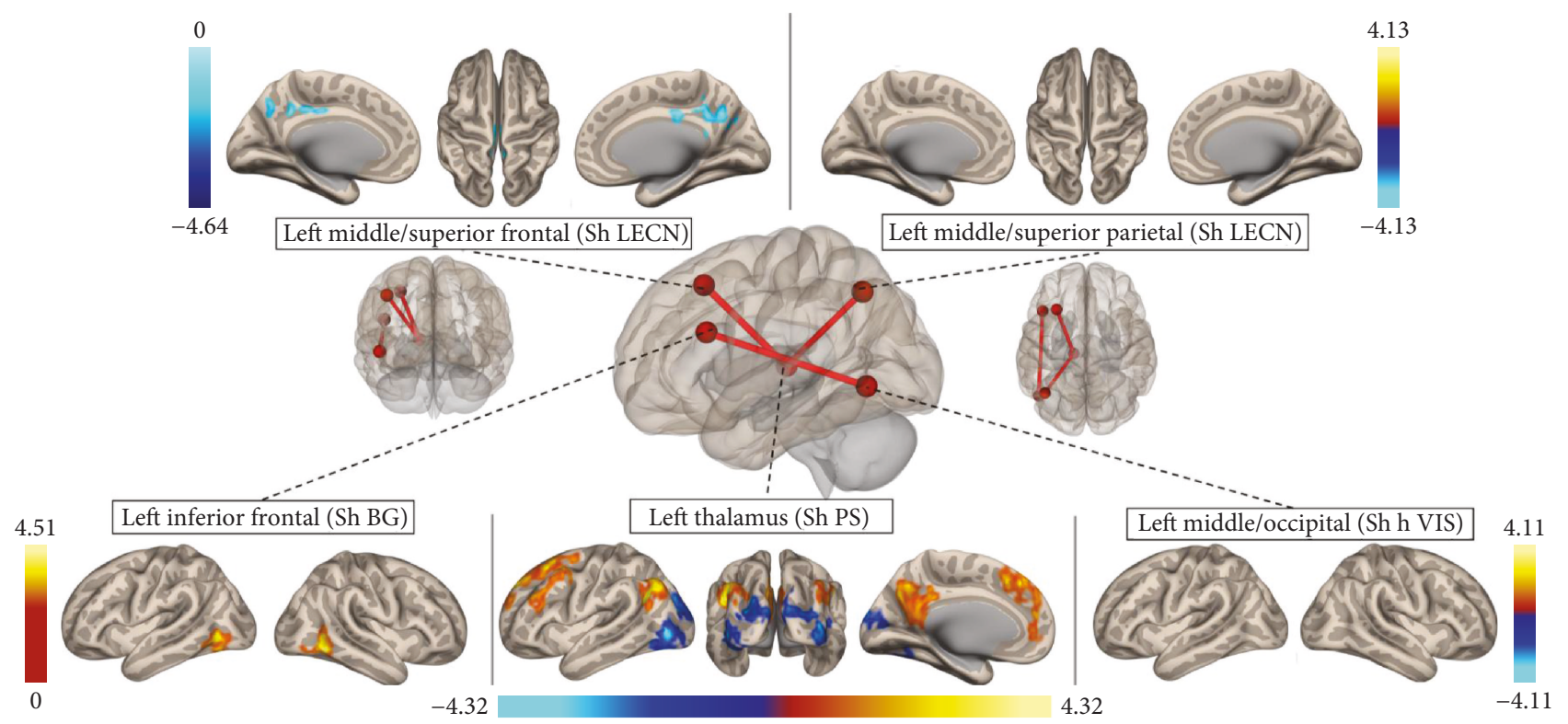

FIGURE 6: Most representative measure of the Power component explained by resting state functional connectivity. ROI-to-ROI analysis reveals a hyperconnectivity in the left inferior frontal-left superior occipital and left superior frontal-left thalamus-left superior parietal connections. Seed-to-voxel results, thresholded (FDR corrected), are shown for each of the 5 nodes. Unthresholded pattern activation is shown in Figure S4 supplementary materials. All voxels with significant connectivity changes are shown in the figure and listed in Table S3, Supplementary materials. Note: H-O: Harvard-Oxford; Sh LECN: Shirer left executive control network; Sh BG: Shirer basal ganglia; Sh PS: Shirer posterior salience; Sh h VIS: Shirer higher visual.

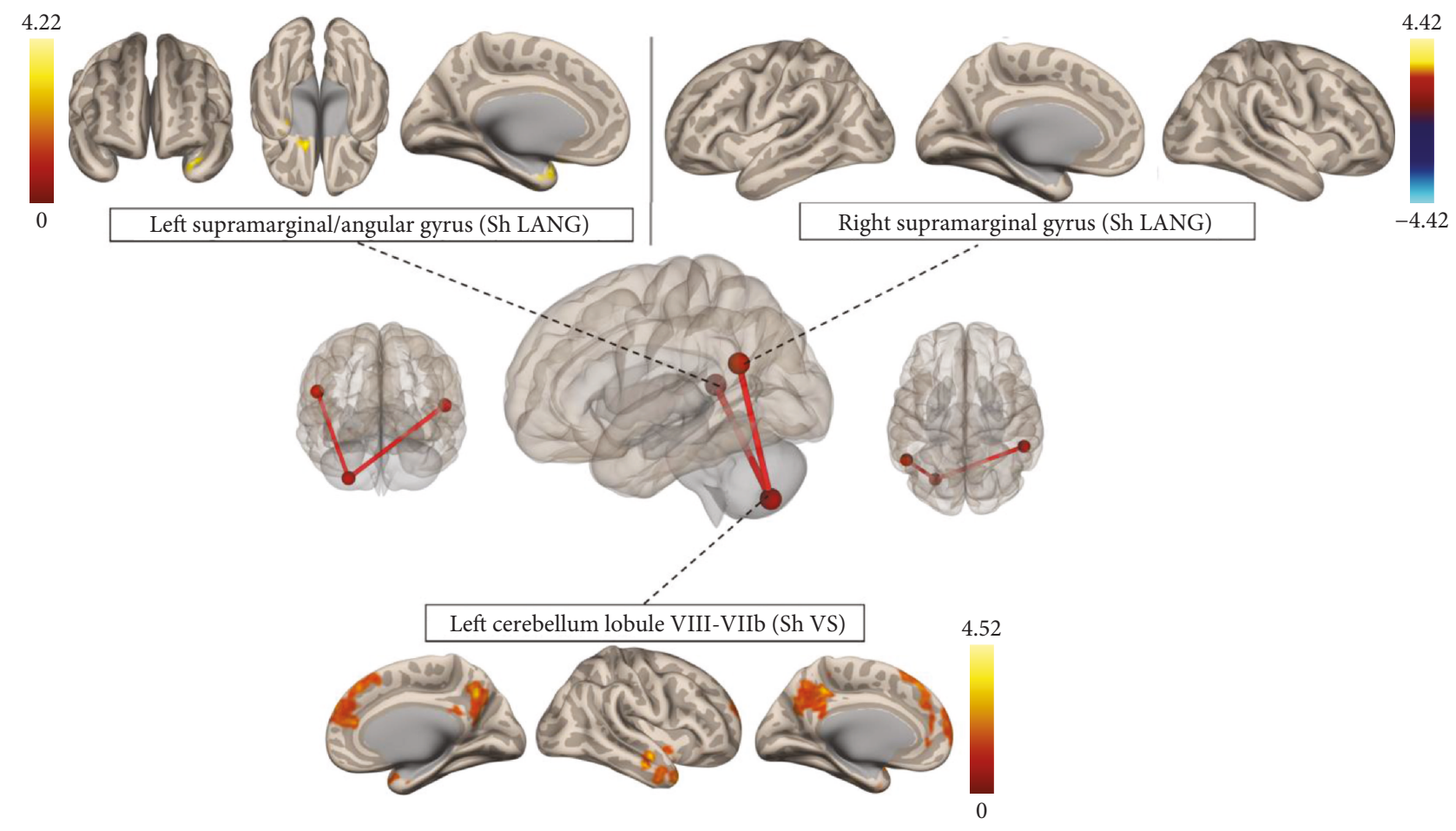

FIGURE 7: Most representative measure of the Speed component explained by resting state functional connectivity. ROI-to-ROI analyses reveal a positive correlation in the cerebellum lobule VIII-VIIb and bilateral superior temporal cortex connections. Seed-to-voxel results, thresholded (FDR corrected), are shown for each of the 3 nodes. Unthresholded pattern activation is shown in Figure S5 supplementary materials. All voxels with significant connectivity changes are shown in the figure and listed in Table S4, Supplementary materials. Note: H-O: Harvard-Oxford; Sh LANG: Shirer language; Sh VS: Shirer visuospatial. 
precuneus $(k=2015 ; 8,-60,40)$, the left angular gyrus $(k=831 ;-54,-56,30)$, and the right $(k=1111 ; 40$, $18,-36)$ and the left $(k=1949 ;-48,-12,-30)$ middle temporal gyrus.

Taking into account the right supramarginal gyrus, seedto-voxel analysis showed an increased connectivity with a cluster of voxels in the cortical and subcortical brain regions $(k=1324 ;-18,0,16)$ such as the left thalamus, left caudate, and left parahippocampal gyrus.

No suprathreshold voxels were found when the right supramarginal gyrus was chosen as the seed.

No significant rs-FC pattern emerged looking at the principal component Execution, which might be due to the weakest relationship with the other variables in the PCA.

\section{Discussion}

Neurophysiological, brain structural, and cognitive behavioural observations have demonstrated positive brain effects following karate training [46-48]. However, the relationships between training-related cognitive and motor skill abilities in karate athletes have not yet been investigated by means of FC. Professional karate is an open skill discipline requiring high-level performance based on explosive and ballistic techniques; therefore, karate is a good example of a competitive sport with high temporal, attentional, and spatial constraints, as it demands rapid RTs, maximal velocity, explosive strength, power, and speed in response to a stimulus. This discipline is divided into kata and kumite. Kata consists of preestablished sequences of offensive and defensive techniques and movements. Kumite, in contrast, is a real match/combat between two competitors under strict rules; they are free to move, kick, and punch in defensive and offensive manners. In both cases, kiai (a Japanese term used in martial arts for the short yell or shout uttered when performing an attacking move) [49] accompanies the feat and expresses the efficacy of the technique: indeed, kiai coincides with the moment when the karateka expresses her/his maximum energy, vigorous application, and perfect timing [49] (see Video 1).

5.1. Cognitive Results in Karateka. In response to the visual search task, we found faster simple RTs in the karate group than in the control group, and ROI-to-ROI connectivity analysis revealed that this difference was sustained by an increase of positive correlations between the right superior parietal lobe and the bilateral occipital poles. These neocortical regions are a stringent part of attentional networks: increased FC in these regions possibly reflects the ability of the karateka to scan the visual environment, identify a previously requested target amongst distractors, and maintain the alert state. This finding is in line with [22], which showed expert golfers to have increased activations in the superior parietal cortex, lateral dorsal premotor cortex, and occipital lobes when they reproduced their motor planning compared with novices, especially during goal-directed movement tasks that are visually triggered [22]. Accordingly, in karate's discipline, the subject has to react as fast as possible to visual (or acoustic, see below) stimuli with maximum speed, power, and control. A high-resolution EEG study of Babiloni and colleagues [15] showed that the parietal and occipital sources of low frequency alpha rhythms $(8-10.5 \mathrm{~Hz})$ were higher in amplitude in the elite karate athletes than in the nonathletes and amateur karate athletes [14]. EEG power is a measure that reflects the capacity or performance of cortical information processing, and the alpha rhythm constitutes an important neural substrate for human cognition and action [50]. In line with this, Babiloni et al. [14, 15] hypothesized that these rhythms would represent a global functional feature that predicts the efficiency of neural cognitive-motor processes when the subject is involved in demanding tasks, and therefore, is in line with our fMRI finding $[14,15]$.

5.2. Kinematic Results of Karateka. On the kinematic side, we asked the karate group to perform 20 frontal punches (gyaku tsuki). RTs, power, and speed of each motor action were recorded and collected by the Ergo-Mak integrated system. $\mathrm{RT}$ is the speed at which a person moves in response to a stimulus, and it represents reactivity of a motor action $[1,2,11]$. We found a positive correlation between "full speed makiwara" (the most representative "Power" feature in the PCA) and a left-lateralized network including the thalamus, inferior and middle frontal gyrus, and superior parietal and middle-occipital cortex. This network might reflect the EEG findings explained with a temporal synchronization of cortical pyramidal neurons by inputs traveling through basal forebrain, thalamus, and cortical feedback loops [14] and might suggest that karateka have the ability to reduce at the minimum their "corticalization" during the execution of motor skills. They, in fact, respond to stimulus with fully ballistic movements, as preprogrammed punches and kicks, that are no longer modifiable during the action [51]. Neuroimaging studies using fMRI have shown that frontoparietal cortical activation is stronger in subjects with high cognitive performance [52], and Babiloni and colleagues [15] showed that elite karate athletes have lower alpha Event-Related Desynchronization (ERD) than controls in parietal and occipital regions during the judgment of karate actions: they interpreted this finding as a possible index of spatially selective cortical activation and in turn of neural efficiency [14]. Furthermore, voxelwise maps have shown a negative correlation between those regions and brain regions (e.g., precuneus) belonging to the default mode networks (DMN). The relevance of negative correlations between DMN and task-positive networks (e.g., frontoparietal control network, anterior salience network, dorsal attention network, and gf-network) in explaining variability in the individual profile [53], response to aging [54], and pathological conditions [55] have been proposed, suggesting this as a pivotal dynamic for brain functioning in general [56]. Here, individuals with faster RTs in karate performance might also display stronger negative correlation between those regions important for the execution of motor skills and the DMN, raising the question about whether a stronger beneficial effect is due to preexisting connectivity and/or cognitive profile. 
Future studies including predefined participants' assignment to a low and a high anticorrelation group are needed to causally test this hypothesis.

Additionally, data showed a positive correlation between "average speed hand" (the most representative "Speed" feature in the PCA) and FC between the cerebellum lobule VIII-VIIb and the bilateral superior temporal cortex. Lesion studies have demonstrated that the cerebellum is critical to the smooth control of movements, and it is also thought to contribute significantly to the generation of forward models, a predictive motor plan that governs the control of ballistic movements [57]. On the other hand, the superior temporal cortex in humans plays a vital role in speech sound processing [58], and environmental sound recognition activates bilateral brain regions including the middle and superior temporal gyrus [59]. In order to succeed in karate, an athlete must locate and identify the relevant perceptual cues, making a decision about what these cues mean, and plan and execute the appropriate motor response accordingly. Oftentimes, all of this happens so quickly that ballistic movements must be initiated with very limited (if any) perceptual information.

Taken together, these finding may unify what has already been shown previously with other neurophysiological approaches, emphasizing the neural correlates of functional characteristics of karate athletes: visuomotor coordination and motor planning of the karateka's technique that must react to external stimuli with ballistics techniques at full speed and power (i.e., noncorticalization of action).

5.3. Audiomotor Cross-Modal Findings. Beyond visual stimuli, either external or internal auditory cues play an important role in guiding karateka's motor performances: these athletes constantly use audible cues like feet stamping, karate-gi (the official dress) slapping or exhalations of the opponent to locate the target and react successfully. Karateka also use constantly their own voice (i.e., the kiai) to accompany the finalization of a given technique.

"Perfect timing" in kumite (see Supplementary materials) entails deciphering small movements (or sounds) and interpreting them as signs of an opponent's decision to launch an attack [60]. It includes the aptitude to perceive those signs and react to them before the attacker is aware of her/his own decision. It also depends on the ability of the brain to automatically select the adequate body motor strategy without access to cognition [60].

Kata is performed alone or in a team. Talking between members of the team is strictly forbidden, so during the performance, audible cues like the exhalation at the end of kime, or rubbing of karate-gi, become fundamental for rhythm and synchronization. Therefore, in karate, audible information is important mostly in defining timing, rhythm, and coordination during the execution, both in kata and kumite performances. During combat, instead, kiai and all the opponent's cues could give anticipatory information for the karateka's attack. We suppose that hearing himself during typical karate sound production could serve as a feedback mechanism in improving karateka technique. We hypothesize that the repetitive exposure to both environmental and self- produced audible cues could well explain the enhanced FC between the cerebellum lobule VIII-VIIb and the bilateral superior temporal cortex: this connectivity profile could be regarded as a new characteristic feature of skillful karateka. Very little is known of the auditory aspects of karate athletes. Woods and colleagues [59] studied with fMRI the neural response to familiar and unfamiliar and sport and nonsport environmental sounds in expert and novice athletes [59]. Experts showed greater activation than novices during the sport familiar condition in regions responsible for auditory and motor planning. The findings that experts showed activation in brain regions known to be involved in action planning when just passively listening to sounds suggest that listening passively to familiar karate sounds like kime, kiai, feet stamping, karate-gi slapping, or exhalation of the opponent could represent an optimal and new indication for training anticipatory skills in karateka.

The importance of the temporal cortex area is highlighted by ROI-to-ROI analysis for rs-fMRI that revealed significant hyperconnectivity in karateka in the left pallidum-right superior temporal gyrus post and right hippocampus-left middle temporal gyrus ant connections compared with the control group. Few neuroimaging studies analysed the expert advantage in sport, especially in the auditory domain; for example, Draganski et al. [61] showed that learning to juggle has been associated with increases in grey matter in a number of areas, with the motion-sensitive middle temporal area increasing bilaterally. Therefore, the auditory channel could represent a suitable training opportunity to improve kinematic skills in karate.

5.4. Limitations of the Study. The control group has been used for comparison of cognitive performances only, while control subjects did not perform any kinematic task. However, functional connectivity analysis in athletes was constrained by specific gestures of the karate discipline that could not be reproduced by subjects of the control group.

It is difficult to say that differences in functional connectivity results are specific for karate athletes, as athletes of other martial arts have not been tested. This should be addressed in future studies.

Moreover, further investigations including larger sample size would be informative about the specificity of the observed functional changes.

\section{Conclusions}

Both cognitive and kinematic behavioural performances have been analysed in professional karate athletes using rs-fMRI as an outcome. Results represent a first step towards the definition of neural correlates sustaining the "power" of karateka's brain in terms of brain efficiency, both in cognitive and motor domains. It is known that structural brain adaptations following extensive training are sport-specific and usually take place in brain regions associated with the neural processing of sport-specific skills [50]. We found an increased positive correlation in the occipital-parietal-temporal network in the karate group, with the left hemisphere more 
prominently involved. This activation pattern likely represents part of the neural correlates of a better performance in attentional tasks in karateka that must be able to take information from their senses (chiefly vision) and transfer it into a motor response to avoid the opponent's attack. Kinematically, we identified increased positive correlations in the bilateral occipital lobe, left thalamus, and left inferior frontal gyrus by analysing the strength parameters of the produced ballistic feat. Finally, increase of connectivity in audiomotor networks, possibly driven by continuous, training-related, external, and internal auditory cues, emerged, suggesting this sensory channel as a possible new training strategy in professional athletes.

\section{Data Availability}

The data used to support the findings of this study are available from the corresponding author upon request.

\section{Conflicts of Interest}

All the authors declare that there is no conflict of interest regarding the publication of this paper.

\section{Authors' Contributions}

Beatrice Berti and Davide Momi contributed equally to this work.

\section{Acknowledgments}

The authors received specific funding from the University of Siena finalized to publication (Piano di Ateneo per la Ricerca 2018).

\section{Supplementary Materials}

Figure S1: positive correlation $\left(R^{2}=65 \%\right)$ between useful field of view accuracies and full speed hand $(\mathrm{m} / \mathrm{s})$. Figure S2: same organization as Figure 4 (main text), but results are shown as unthresholded maps. Figure S3: same organization as Figure 5 (main text), but results are shown as unthresholded maps. Figure S4: same organization as Figure 6 (main text), but results are shown as unthresholded maps. Figure S5: same organization as Figure 7 (main text), but results are shown as unthresholded maps. Table S1: seedto-voxel-based analysis of significant clusters that showed a significant increase (red arrow) or decrease (blue arrow) correlation in the karate group. Table S2: seed-to-voxel-based analysis of significant clusters that showed a significant increase (red arrow) or decrease (blue arrow) correlation for visual search reaction time performance. Table S3: seed-to-voxel-based analysis of significant clusters that showed a significant increase (red arrow) or decrease (blue arrow) correlation for the most representative measure of the Power component. Table S4: seed-to-voxel-based analysis of significant clusters that showed a significant increase (red arrow) or decrease (blue arrow) correlation for the most representative measure of the Speed component. (Supplementary Materials)

\section{References}

[1] S. Mori, Y. Ohtani, and K. Imanaka, "Reaction times and anticipatory skills of karate athletes," Human Movement Science, vol. 21, no. 2, pp. 213-230, 2002.

[2] G. Fontani, S. Migliorini, R. Benocci, A. Facchini, M. Casini, and F. Corradeschi, "Effect of mental imagery on the development of skilled motor actions," Perceptual and Motor Skills, vol. 105, no. 3, pp. 803-826, 2007.

[3] G. Fontani, L. Lodi, A. Felici, S. Migliorini, and F. Corradeschi, "Attention in athletes of high and low experience engaged in different open skill sports," Perceptual and Motor Skills, vol. 102, no. 3, pp. 791-805, 2006.

[4] K. Witte, S. Kropf, S. Darius, P. Emmermacher, and I. Böckelmann, "Comparing the effectiveness of karate and fitness training on cognitive functioning in older adults-a randomized controlled trial," Journal of Sport and Health Science, vol. 5, no. 4, pp. 484-490, 2016.

[5] G. Ravier, F. Grappe, and J. D. Rouillon, "Application of forcevelocity cycle ergometer test and vertical jump tests in the functional assessment of karate competitor, ResearchGate, July 2017, https://www.researchgate.net/publication/ 7975251_Application_of_force-velocity_cycle_ergometer_ test_and_vertical_jump_tests_in_the_functional_assess ment_of_karate_competitor.

[6] E. P. Zehr, D. G. Sale, and J. J. Dowling, "Ballistic movement performance in karate athletes," Medicine and Science in Sports and Exercise, vol. 29, no. 10, pp. 1366-1373, 1997.

[7] C. Doria, A. Veicsteinas, E. Limonta et al., "Energetics of karate (kata and kumite techniques) in top-level athletes," European Journal of Applied Physiology, vol. 107, no. 5, pp. 603-610, 2009.

[8] H. Roschel, M. Batista, R. Monteiro et al., "Association between neuromuscular tests and kumite performance on the Brazilian Karate National Team," Journal of Sports Science and Medicine, vol. 8, no. CSSI3, pp. 20-24, 2009.

[9] N. Koropanovski, B. Berjan, P. Bozic et al., "Anthropometric and physical performance profiles of elite karate kumite and kata competitors," Journal of Human Kinetics, vol. 30, no. 1, pp. 107-114, 2011.

[10] J. Pozo, G. Bastien, and F. Dierick, "Execution time, kinetics, and kinematics of the mae-geri kick: comparison of national and international standard karate athletes," Journal of Sports Sciences, vol. 29, no. 14, pp. 1553-1561, 2011.

[11] H. Chaabène, Y. Hachana, E. Franchini, B. Mkaouer, and K. Chamari, "Physical and physiological profile of elite karate athletes," Sports Medicine, vol. 42, no. 10, pp. 829-843, 2012.

[12] C. Del Percio, C. Babiloni, N. Marzano et al., "“Neural efficiency" of athletes' brain for upright standing: a highresolution EEG study," Brain Research Bulletin, vol. 79, no. 3-4, pp. 193-200, 2009.

[13] C. Del Percio, F. Infarinato, M. Iacoboni et al., "Movementrelated desynchronization of alpha rhythms is lower in athletes than non-athletes: a high-resolution EEG study," Clinical Neurophysiology, vol. 121, no. 4, pp. 482-491, 2010.

[14] C. Babiloni, N. Marzano, F. Infarinato et al., "“Neural efficiency" of experts' brain during judgment of actions: a highresolution EEG study in elite and amateur karate athletes," Behavioural Brain Research, vol. 207, no. 2, pp. 466-475, 2010.

[15] C. Babiloni, N. Marzano, M. Iacoboni et al., "Resting state cortical rhythms in athletes: a high-resolution EEG study," Brain Research Bulletin, vol. 81, no. 1, pp. 149-156, 2010. 
[16] R. E. Roberts, P. G. Bain, B. L. Day, and M. Husain, "Individual differences in expert motor coordination associated with white matter microstructure in the cerebellum," Cerebral Cortex, vol. 23, no. 10, pp. 2282-2292, 2013.

[17] B. Dunst, M. Benedek, E. Jauk et al., "Neural efficiency as a function of task demands," Intelligence, vol. 42, no. 100, pp. 22-30, 2014.

[18] R. J. Haier, B. Siegel, C. Tang, L. Abel, and M. S. Buchsbaum, "Intelligence and changes in regional cerebral glucose metabolic rate following learning," Intelligence, vol. 16, no. 3-4, pp. 415-426, 1992.

[19] V. Charlot, N. Tzourio, M. Zilbovicius, B. Mazoyer, and M. Denis, "Different mental imagery abilities result in different regional cerebral blood flow activation patterns during cognitive tasks," Neuropsychologia, vol. 30, no. 6, pp. 565-580, 1992.

[20] B. Rypma, J. Berger, H. Genova, D. Rebbechi, and M. Desposito, "Dissociating age-related changes in cognitive strategy and neural efficiency using event- related fMRI," Cortex, vol. 41, no. 4, pp. 582-594, 2005.

[21] C. C. Ruff, M. Knauff, T. Fangmeier, and J. Spreer, "Reasoning and working memory: common and distinct neuronal processes," Neuropsychologia, vol. 41, no. 9, pp. 1241-1253, 2003.

[22] J. Milton, A. Solodkin, P. Hlustík, and S. L. Small, "The mind of expert motor performance is cool and focused," NeuroImage, vol. 35, no. 2, pp. 804-813, 2007.

[23] Y.-T. Kim, J.-H. Seo, H.-J. Song et al., "Neural correlates related to action observation in expert archers," Behavioural Brain Research, vol. 223, no. 2, pp. 342-347, 2011.

[24] G. Bernardi, E. Ricciardi, L. Sani et al., "How skill expertise shapes the brain functional architecture: an fMRI study of visuo-spatial and motor processing in professional racing-car and naïve drivers," PLoS One, vol. 8, no. 10, article e77764, 2013.

[25] E. Naito and S. Hirose, "Efficient foot motor control by Neymar's brain," Frontiers in Human Neuroscience, vol. 8, p. 594, 2014.

[26] M. Lotze, G. Scheler, H.-R. M. Tan, C. Braun, and N. Birbaumer, "The musician's brain: functional imaging of amateurs and professionals during performance and imagery," NeuroImage, vol. 20, no. 3, pp. 1817-1829, 2003.

[27] A. Sekiguchi, S. Yokoyama, S. Kasahara et al., "Neural bases of a specific strategy for visuospatial processing in rugby players," Medicine and Science in Sports and Exercise, vol. 43, no. 10, pp. 1857-1862, 2011.

[28] J. Seo, Y.-T. Kim, H.-J. Song et al., "Stronger activation and deactivation in archery experts for differential cognitive strategy in visuospatial working memory processing," Behavioural Brain Research, vol. 229, no. 1, pp. 185-193, 2012.

[29] B. Biswal, F. Zerrin Yetkin, V. M. Haughton, and J. S. Hyde, "Functional connectivity in the motor cortex of resting human brain using echo-planar MRI," Magnetic Resonance in Medicine, vol. 34, no. 4, pp. 537-541, 1995.

[30] M. J. Lowe, B. J. Mock, and J. A. Sorenson, "Functional connectivity in single and multislice echoplanar imaging using resting-state fluctuations," NeuroImage, vol. 7, no. 2, pp. 119-132, 1998.

[31] D. Cordes, V. M. Haughton, K. Arfanakis et al., "Mapping functionally related regions of brain with functional connectivity MR imaging," American Journal of Neuroradiology, vol. 21, no. 9, pp. 1636-1644, 2000.
[32] M. Hampson, B. S. Peterson, P. Skudlarski, J. C. Gatenby, and J. C. Gore, "Detection of functional connectivity using temporal correlations in MR images," Human Brain Mapping, vol. 15, no. 4, pp. 247-262, 2002.

[33] M. D. Greicius, B. Krasnow, A. L. Reiss, and V. Menon, "Functional connectivity in the resting brain: a network analysis of the default mode hypothesis," Proceedings of the National Academy of Sciences, vol. 100, no. 1, pp. 253-258, 2003.

[34] H. Laufs, K. Krakow, P. Sterzer et al., "Electroencephalographic signatures of attentional and cognitive default modes in spontaneous brain activity fluctuations at rest," Proceedings of the National Academy of Sciences of the United States of America, vol. 100, no. 19, pp. 11053-11058, 2003.

[35] V. G. van de Ven, E. Formisano, D. Prvulovic, C. H. Roeder, and D. E. J. Linden, "Functional connectivity as revealed by spatial independent component analysis of fMRI measurements during rest," Human Brain Mapping, vol. 22, no. 3, pp. 165-178, 2004.

[36] M. Greicius, "Resting-state functional connectivity in neuropsychiatric disorders," Current Opinion in Neurology, vol. 24, no. 4, pp. 424-430, 2008.

[37] M. D. Fox and M. Greicius, "Clinical applications of resting state functional connectivity," Frontiers in Systems Neuroscience, vol. 4, p. 19, 2010.

[38] D. Zhang and M. E. Raichle, "Disease and the brain's dark energy," Nature Reviews. Neurology, vol. 6, no. 1, pp. 15-28, 2010.

[39] M. D. Fox, R. L. Buckner, H. Liu, M. M. Chakravarty, A. M. Lozano, and A. Pascual-Leone, "Resting-state networks link invasive and noninvasive brain stimulation across diverse psychiatric and neurological diseases," Proceedings of the National Academy of Sciences of the United States of America, vol. 111, no. 41, pp. E4367-E4375, 2014.

[40] D. Salmaso and A. M. Longoni, "Hand preference in an Italian sample," Perceptual and Motor Skills, vol. 57, 3_Supplement, pp. 1039-1042, 1983.

[41] IBM Corp, BM SPSS Statistics for Windows, Version 20.0, IBM Corp, Armonk, NY, 2011.

[42] S. Wold, K. Esbensen, and P. Geladi, "Principal component analysis," Chemometrics and Intelligent Laboratory Systems, vol. 2, no. 1-3, pp. 37-52, 1987.

[43] S. Whitfield-Gabrieli and A. Nieto-Castanon, "Conn: a functional connectivity toolbox for correlated and anticorrelated brain networks," Brain Connectivity, vol. 2, no. 3, pp. 125-141, 2012.

[44] J. Chumbley, K. Worsley, G. Flandin, and K. Friston, “Topological FDR for neuroimaging," NeuroImage, vol. 49, no. 4, pp. 3057-3064, 2010.

[45] A. Zalesky, A. Fornito, and E. T. Bullmore, "Network-based statistic: identifying differences in brain networks," NeuroImage, vol. 53, no. 4, pp. 1197-1207, 2010.

[46] K. D. Conant, A. K. Morgan, D. Muzykewicz, D. C. Clark, and E. A. Thiele, "A karate program for improving self-concept and quality of life in childhood epilepsy: results of a pilot study," Epilepsy \& Behavior, vol. 12, no. 1, pp. 61-65, 2008.

[47] C.-D. Marie-Ludivine, G. Papouin, P. Saint-Val, and A. Lopez, "Effect of adapted karate training on quality of life and body balance in 50-year-old men," Open access Journal of Sports Medicine, vol. 1, pp. 143-150, 2010.

[48] P. Jansen and K. Dahmen-Zimmer, "Effects of cognitive, motor, and karate training on cognitive functioning and 
emotional well-being of elderly people," Frontiers in Psychology, vol. 3, p. 40, 2012.

[49] M. Daimaruya, H. Fujiki, and H. Anbarita, "Consideration of the power in karate-doh from the viewpoint of impact engineering," 2011, July 2017, http://docplayer.net/ 45945530-Consideration-of-the-power-in-karate-doh-fromthe-viewpoint-of-impact-engineering.html.

[50] W. Klimesch, M. Doppelmayr, J. Schwaiger, P. Auinger, and T. Winkler, "“Paradoxical” alpha synchronization in a memory task," Brain Research. Cognitive Brain Research, vol. 7, no. 4, pp. 493-501, 1999.

[51] D. T. McMaster, N. Gill, J. Cronin, and M. McGuigan, “A brief review of strength and ballistic assessment methodologies in sport," Sports Medicine, vol. 44, no. 5, pp. 603-623, 2014.

[52] S. D. Newman, P. A. Carpenter, S. Varma, and M. A. Just, "Frontal and parietal participation in problem solving in the Tower of London: fMRI and computational modeling of planning and high-level perception," Neuropsychologia, vol. 41, no. 12 , pp. $1668-1682,2003$.

[53] R. N. Spreng, J. Sepulcre, G. R. Turner, W. D. Stevens, and D. L. Schacter, "Intrinsic architecture underlying the relations among the default, dorsal attention, and frontoparietal control networks of the human brain," Journal of Cognitive Neuroscience, vol. 25, no. 1, pp. 74-86, 2013.

[54] R. N. Spreng, W. D. Stevens, J. D. Viviano, and D. L. Schacter, "Attenuated anticorrelation between the default and dorsal attention networks with aging: evidence from task and rest," Neurobiology of Aging, vol. 45, pp. 149-160, 2016.

[55] J. Zhou, M. D. Greicius, E. D. Gennatas et al., "Divergent network connectivity changes in behavioural variant frontotemporal dementia and Alzheimer's disease," Brain, vol. 133, no. 5, pp. 1352-1367, 2010.

[56] M. D. Fox, A. Z. Snyder, J. L. Vincent, M. Corbetta, D. C. van Essen, and M. E. Raichle, "The human brain is intrinsically organized into dynamic, anticorrelated functional networks," Proceedings of the National Academy of Sciences of the United States of America, vol. 102, no. 27, pp. 9673-9678, 2005.

[57] D. M. Wolpert and R. C. Miall, "Forward models for physiological motor control," Neural Networks, vol. 9, no. 8, pp. 1265-1279, 1996.

[58] J. R. Binder, J. A. Frost, T. A. Hammeke et al., "Human temporal lobe activation by speech and nonspeech sounds," Cerebral Cortex, vol. 10, no. 5, pp. 512-528, 2000.

[59] E. A. Woods, A. E. Hernandez, V. E. Wagner, and S. L. Beilock, "Expert athletes activate somatosensory and motor planning regions of the brain when passively listening to familiar sports sounds," Brain and Cognition, vol. 87, pp. 122-133, 2014.

[60] E. B.-O. Cohen, "Timing in karate and the body in its own right," Social Analysis, vol. 51, no. 3, pp. 1-22, 2007.

[61] B. Draganski, C. Gaser, V. Busch, G. Schuierer, U. Bogdahn, and A. May, "Neuroplasticity: changes in grey matter induced by training," Nature, vol. 427, no. 6972, pp. 311-312, 2004. 


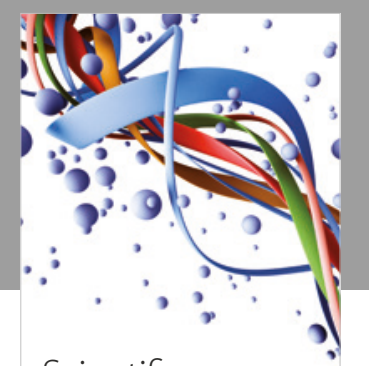

Scientifica
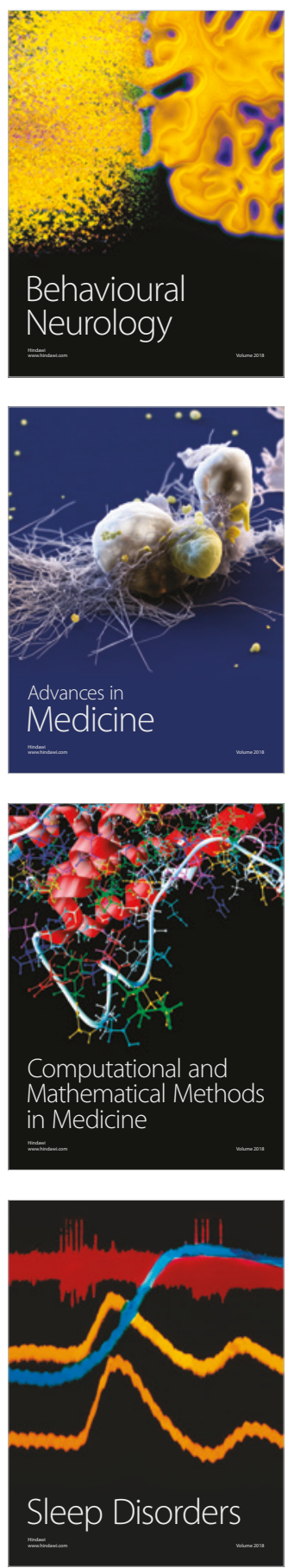

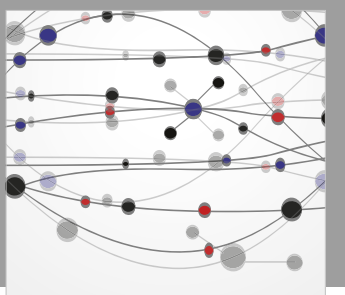

The Scientific World Journal

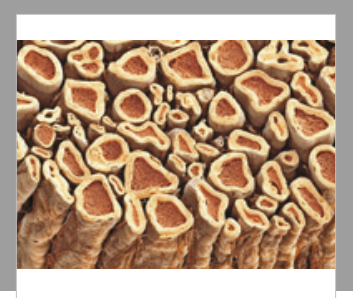

Case Reports in

Neurological Medicine

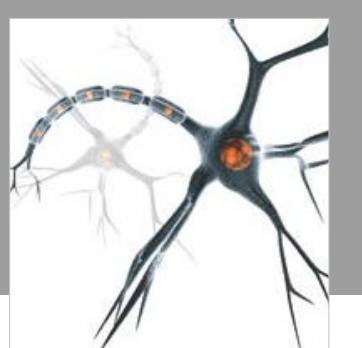

Neural Plasticity

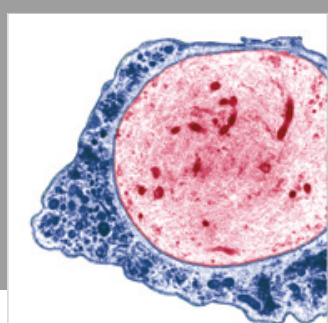

Multiple Sclerosis

International

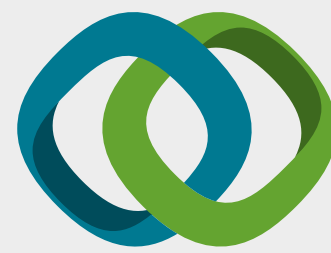

Hindawi

Submit your manuscripts at

www.hindawi.com
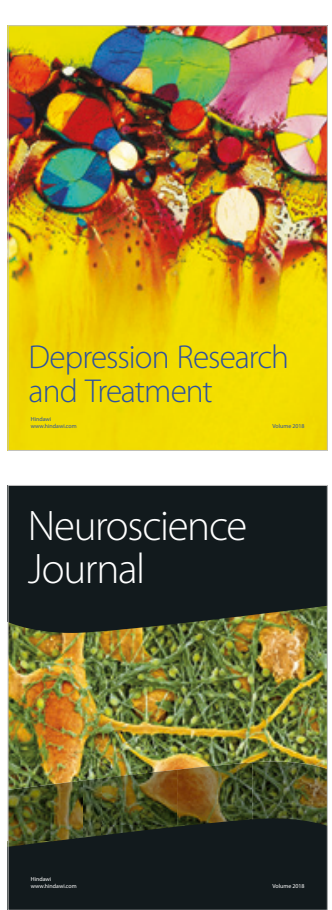

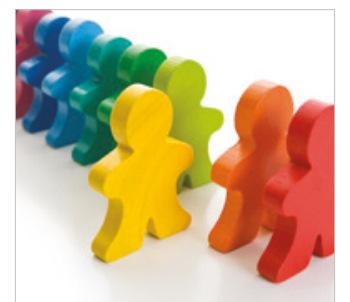

Autism

Research and Treatment

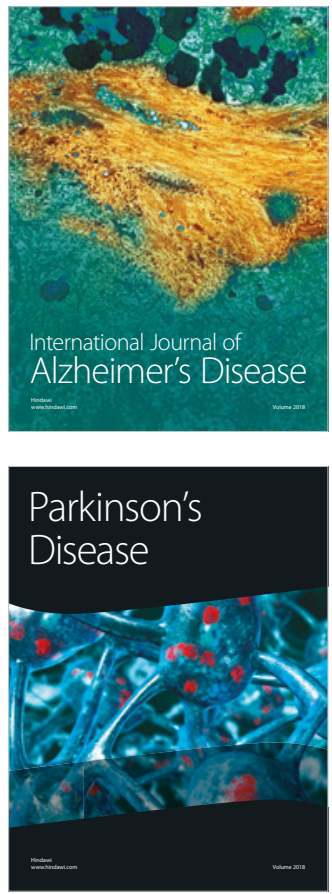
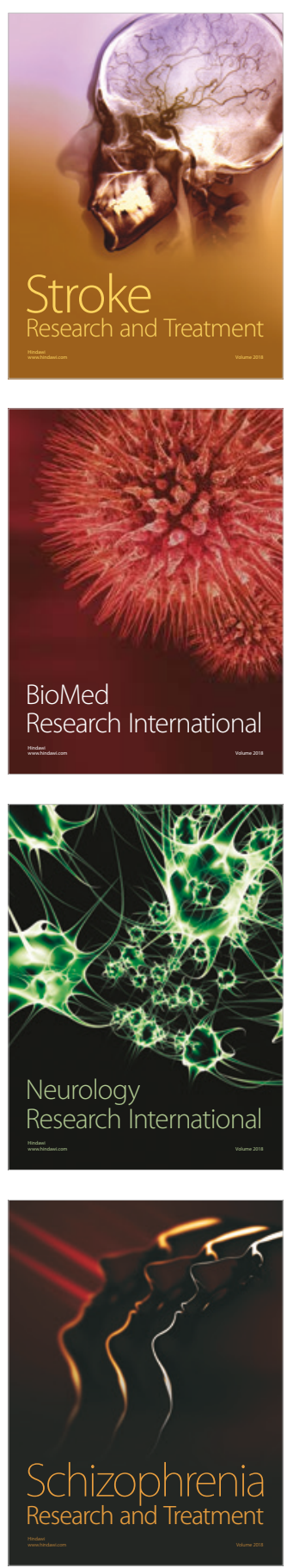